\title{
The information highway between conceptus and soma: when conversations get confused
}

\author{
David F. Albertini ${ }^{1}$ \\ Published online: 18 February 2019 \\ (C) Springer Science+Business Media, LLC, part of Springer Nature 2019
}

Carrying out conversations between distinct cell or tissue types is a hallmark of systems biology. Both the nature of the language itself - the kinds of chemical signals deployed - and the means by which a signal is received and acted upon, constitute the framework upon which reproductive competence is realized. Of the multitude of conversational interfaces at work within organisms of the viviparous variety (those of the live young-bearing ilk), none is more important than the interface between the embryo and uterine endometrium in species exhibiting implantation of either a non-invasive or invasive character. This month we feature reviews that take a close look at some of the physico-chemical mediators of communication between conceptus and soma (The role of microRNAs in human embryo implantation: a review, https://oi.org/10.1007/s10815-018-1326), and a type of vesicle that has become a dominant player on the implantation stage (Extracellular vesicle mediated embryoendometrial cross talk during implantation and in pregnancy, https://doi.org/10.1007/s10815-018-1343).

Exosomes have taken center stage as communication devices at many loci within the mammalian reproductive tract. They serve as a prominent feature of the microenvironment mediating embryo-endometrium interactions [1], and those derived from the endometrium have been recognized for several years now to impart upon trophectoderm alterations in adhesivity that contribute in form and substance to the attachment of the embryo to the uterine epithelium in anticipation of an attempt to implant [2]. Over and above defining when exosomes are produced to influence their assigned targets, what cargo content is mediating changes in gene expression or target cell responsiveness has become a legitimate focus of study under the purview of infertility affecting men or women.

David F. Albertini

eicjarg@gmail.com

1 Center for Human Reproduction, New York, NY, USA
Some readers may recall another scenario, reported recently in JARG, within the epididymis where exosomes from the epithelium seem to confer enhanced fertilizing capacity upon sperm [3]. Studies of this kind continue to reinforce the growing notion that gametes and embryos are in receipt of vital cues from the soma via the contents born within vesicles. Timely positional stickiness may be one central determinant for successful implantation. But, as work over the past years has shown, human embryos have an extraordinary selforganizing capability that must in some fashion be harnessed or modulated by signals emanating from the uterine epithelium.

To wit, our cover this month reflects the intrinsic ability of the trophectoderm to initiate and sustain not only adhesion to an unnatural substrate, but an ability to carry out programs necessary to specify fates for cellular lineages within and without the epiblast, as shown in earlier studies $[4,5]$. These dramatic events unfold outside of a uterine milieu, as do the morphogenetic gymnastics of compaction and blastocyst expansion routinely visualized in the daily practice of human ARTs. While genetic imbalances in part can influence both the kinetics and normalcy of preimplantation development (Time-lapse imaging reveals delayed development of embryos carrying unbalanced chromosomal translocations, https://doi. org/10.1007/s10815-018-1361), the often implicated deleterious role of reactive oxygen species expected under the culture conditions commonly used in ART seems not to be as likely a culprit as previously thought (Limited relationships between reactive oxygen species levels in culture media and zygote and embryo development, https:// doi.org/10.1007/s10815-018-1363). How then will the separation of powers between embryo and soma be sorted out?

One long-held notion takes as a point of departure the bioenergetic demands imposed on the embryo as it transits to the site of implantation, and perhaps well beyond in terms of autonomy. Interestingly, while attention continues to be focused on ATP metabolism within the embryo itself, the study 
of May-Panloup and colleagues raises the interesting prospect that where mtDNA comes into the picture (if indeed it is a sentinel of anything to do with driving early development) of embryo developmental competence is not after fertilization but before (The mitochondrial DNA content of cumulus cells may help predict embryo implantation, https://doi.org/10. 1007/s10815-018-1348). Should these findings be confirmed and extended, the possibility exists that the embryo's allocation of maternally inherited mitochondria will become the distractor at the expense of what the embryo is endowed with, again from somatic elements of the ovary.

While no answers are immediately forthcoming as the effort to resolve relative contributions of conceptus and soma at the time of implantation, there is light on the horizon. Like light sheet microscopy, insights into the critical morphogenetic events taking place within the embryo at the time of implantation were first demonstrated using light sheet microscopy on preimplantation mouse embryos, as the work of Ellenberg and colleagues so elegantly demonstrated [6]. The next great advance, again using the postimplantation mouse embryo, has arrived in the remarkable studies of McDole et al. reported late in 2018 [7]. These advances in biomedical imaging, long having come to fruition at the level of the whole organism, are now clearly pushing the limits of what we will see at a single-cell level as that dialog between mom and embryo plays out during implantation.
Publisher's note Springer Nature remains neutral with regard to jurisdictional claims in published maps and institutional affiliations.

\section{References}

1. Salamonsen LA, Evans J, Nguyen HP, Edgell TA. The microenvironment of human implantation: determinant of reproductive success. Am J Reprod Immunol. 2016;75(3):218-25.

2. Greening DW, Nguyen HP, Elgass K, Simpson RJ, Salamonsen LA. Human endometrial exosomes contain hormone-specific cargo modulating trophoblast adhesive capacity: insights into endometrialembryo interactions. Biol Reprod. 2016;94(2):38.

3. Rowlison T, Ottinger MA, Comizzoli P. Key factors enhancing sperm fertilizing ability are transferred from the epididymis to the spermatozoa via epididymosomes in the domestic cat model. J Assist Reprod Genet. 2018;35(2):221-8.

4. Deglincerti A, Croft GF, Pietila LN, Zernicka-Goetz M, Siggia ED, Brivanlou AH. Self-organization of the in vitro attached human embryo. Nature. 2016;533(7602):251-4.

5. Shahbazi MN, Jedrusik A, Vuoristo S, Recher G, Hupalowska A. Bolton V, et al. Nat Cell Biol: Self-organization of the human embryo in the absence of maternal tissues; 2016.

6. Strnad P, Gunther S, Reichmann J, Krzic U, Balazs B, de Medeiros $\mathrm{G}$, et al. Inverted light-sheet microscope for imaging mouse preimplantation development. Nat Methods. 2016;13(2):139-42.

7. McDole K, Guignard L, Amat F, Berger A, Malandain G, Royer LA, et al. In toto imaging and reconstruction of post-implantation mouse development at the single-cell level. Cell. 2018;175(3):859-76 e33. 